\title{
Schöne Neue Welt..., wer blickt noch durch?
}

Das Werk von Aldous Huxley ist sicher noch manchem Leser bekannt. Doch was in den letzten Jahren an rasanter Entwicklung gelaufen ist, war selbst in den Tagen dieses genialen Schriftstellers jenseits des Vorstellbaren: Gentechnik, Internet, denkende Roboter, Mobile Computing, 3D-Visualisierung, Datenaustausch durch sekundenschnelle weltweite Vernetzung... Die Kommunikation erfordert immer mehr statt weniger Zeit.

Viele dieser neuen Felder rauben uns den Atem, sind für den Laien - ähnlich wie die Kernkraft - bis heute in ihrer Funktionsweise nicht verständlich. Dies macht uns misstrauisch, unsicher. Um so mehr gilt es selbstregulierende und -bewertende Instrumente auszubauen, die verhindern, dass sich eines Tages ein derartiger Zweig autark und jenseits des Gemeinwohls entwickelt.

In der Medizin stellt das rasante Anwachsen des Wissens sowohl Forscher als auch Verlage und Leser vor immer neue Herausforderungen. Das Forschen nimmt mittlerweile deutlich weniger Zeit in Anspruch wie die Vorbereitung der entsprechenden Publikation, deren Begutachtung und insbesondere die Beantragung von Forschungsgeldern - sei es auf nationalem, europäischem oder internationalem Level. Die Drucklegung erfolgt z.T. mit deutlicher zeitlicher Verzögerung und ist in kompetitiven Feldern mittlerweile schon ein richtiges Ärgernis. Das Internet ist im Bereich der Fachinformation als Instrument des Wissensmanagements deutlich im Vormarsch. Es gehört nicht viel Phantasie zu der Vorstellung, Publikationen online einzureichen, sie elektronisch Gutachtern vorzustellen und sie sodann in eine Datenbank mit Volltextindizierung einzubinden.

\section{Was bietet zukünftig eine Zeitschrift wie die „Aktuelle Dermatologie“?}

Sie ist prädestiniert für praxisrelevante Artikel aus der dermatologisch-klinisch orientierten zunehmend biomedizinisch ausgerichteten Forschung, für gut aufgemachte Übersichten zu praxisrelevanten Themenfeldern (Themenhefte), Diskussion von fachspezifisch relevanten „Leitlinien“, wichtige Mit- teilungen der Verbände und der Industrie und natürlich für den Blick über den eigenen Zaun hin zu neuen Entwicklungen - von der IT-Industrie über Mediziner-relevantes Managementwissen bis hin zu neuen Therapieverfahren z.B. in der Inneren Medizin, die für uns möglicherweise wegen potenzieller Nebenwirkungen von Interesse sind. Der fachübergreifend operierende Thieme Verlag ist hier ein idealer Partner. Hinterlegt der Verlag die Zeitschrift mit einer entsprechenden Online-Datenbank, ist der Nutzen für den Abonnenten rund um die Uhr gegeben. Spannend wird dann die Quervernetzung zu weiteren Themenfeldern.

Was wir daher unbedingt benötigen sind Sie, verehrter Leser! Nur Ihre aktive Mitarbeit gestattet es uns, Ihnen - gewissermaßen basisdemokratisch - die Zeitschrift und den Service zu liefern, die Ihren Ansprüchen gerecht wird. Daher - schreiben Sie uns, wenn Sie Vorschläge für neue Themenfelder, andere Aufmachung oder einfach konstruktive Kritik haben. Die Herausgeber nehmen Ihre Anregungen ernst und diskutieren sie intern und mit dem Verlag.

Die Zukunft liegt im sogenannten Cross Media Publishing - für jeden Nutzerkreis zu jedem Zweck das richtige Medium. So können Sie - als Niedergelassener oder als Kliniker - Artikel hoffentlich bald via Internetformular z.B. auf die Praxisrelevanz hin bewerten, ein wichtiger und richtiger Schritt hin zur Sicherung von Behandlungsqualität; denn Artikel mit der höchsten Relevanz sollten alle Kollegen gelesen und verstanden haben.

Die Zukunft heißt denn auch: Gesundheitsinformation als Mittel des freiwilligen ärztlichen Qualitätsmanagements!

Schöne Neue Welt... doch gar nicht so verkehrt!?!

Zum Nachdenken und Weiterentwickeln!

M. Herbst, Darmstadt 